\section{Cahiers de Narratologie}

Analyse et théorie narratives

$14 \mid 2008$

Prose d'idées : formes et savoirs

\title{
Edmond CROS, Le sujet culturel, sociocritique et psychanalyse, Paris, L'Harmattan, 2005, 270 p.
}

\section{Marc Marti}

\section{(2) OpenEdition}

Journals

Édition électronique

URL : http://journals.openedition.org/narratologie/597

DOI : 10.4000/narratologie.597

ISSN : 1765-307X

Éditeur

LIRCES

\section{Référence électronique}

Marc Marti, «Edmond cros, Le sujet culturel, sociocritique et psychanalyse, Paris, L'Harmattan, 2005,

270 p. », Cahiers de Narratologie [En ligne], 14 | 2008, mis en ligne le 15 juillet 2010, consulté le 22 septembre 2020. URL : http://journals.openedition.org/narratologie/597 ; DOI : https://doi.org/ 10.4000/narratologie.597

Ce document a été généré automatiquement le 22 septembre 2020.

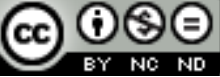

Cahiers de Narratologie - Analyse et théorie narratives est mis à disposition selon les termes de la licence Creative Commons Attribution - Pas d'Utilisation Commerciale - Pas de Modification 4.0 International. 


\title{
Edmond CROS, Le sujet culturel, sociocritique et psychanalyse, Paris, L'Harmattan, 2005, 270 p.
}

\author{
Marc Marti
}

1 Née dans les années soixante au croisement de la psychanalyse et du matérialisme dialectique, la sociocritique s'est donné pour objectif un renouvellement de l'approche sociologique de la littérature qui intègrerait les avancées du structuralisme, de la sémiologie et de la linguistique. Edmond Cros, co-fondateur de la discipline en France avec Claude Duchet, propose - après La sociocritique, L'Harmattan, 2003 http:// revel.unice.fr/cnarra/document.html?id=33 - un second ouvrage de synthèse plus particulièrement centré sur la question du sujet culturel. Ce livre, comme le précédent, est le produit d'une pratique de plus de trente années, tant comme chercheur dans le cadre de l'ISS (Institut International de Sociocritique) que comme enseignant à l'Université de Montpellier.

2 L'ouvrage se présente en quinze chapitres et quatre parties. La première partie, intitulée "D'un sujet à l'autre ", pose un essai de définition et de fonctionnement du sujet culturel, et renvoie, hormis les références à la sociocritique, principalement aux travaux de Benveniste et aux écrits de Jacques Lacan. Pour la sociocritique, le problème de départ passe par la définition et la nature du sujet. Je ou moi, selon les termes établis en fonction de la position du sujet, la question reste « quelle est la part du nous dans ce je ou dans ce moi? Quelle est la part de l'autre dans ce moi ou dans ce je? Rappelant la définition de "sujet transindividuel ", forgée par Lucien Goldmann et qui appelle le concept de "non-conscient», qui se différencie de l'inconscient par l'absence de refoulement, Edmond Cros propose de définir ensuite le «sujet culturel », une notion recouvrant sans s'y superposer exactement le sujet idéologique et le sujet transindividuel. L'intérêt de la notion est qu'elle permet de prendre en compte la dimension individuelle et collective. Le sujet culturel, en tant que système « s'organise autour d'un élément dominant qui restera potentiellement dominant jusqu'à ce qu'intervienne une altération significative de l'infrastructure ou, plus exactement, 
jusqu'à ce que celle-ci soit répercutée dans le champ discursif et plus largement dans le champ culturel ». On peut cependant affiner la notion de sujet transindividuel de Goldmann, en rappelant que celui-ci est chargé de temps historiques multiples qui coexistent. Le sujet culturel est examiné sous l'angle du rapport au langage et de son appropriation. Le sujet culturel est à la fois au croisement de la formation de la subjectivité et des processus de socialisation. Se fondant sur le stade du miroir chez Lacan, l'auteur souligne que le sujet ne s'identifie pas au modèle culturel, c'est ce dernier qui le fait advenir comme sujet. Le sujet culturel se retrouve donc à l'articulation de trois formations -sociales, idéologiques et discursives-, qui possèdent leur propre rythme d'existence, induisant phasages et déphasages successifs en fonction des époques et des lieux. La partie se clôt par une analyse d'application au starsystem.

3 La seconde partie «Les modes d'inscription de l'idéologique et de l'histoire dans la compétence sémiotique du sujet " reprend un certain nombre de définitions déjà formulées par l'auteur dans des travaux antérieurs, en particulier celle du texte sémiotique et de l'idéosème. Pour Edmond Cros, l'établissement du système sémiotique consiste à s'intéresser non pas à la convergence des signes pour ce qu'ils expriment mais pour ce qu'ils sont. Il ne s'agit pas de s'intéresser à la participation du signe au sens de l'énoncé mais «à ce qu'il signifie, en liaison avec d'autres signes, indépendamment de ce que dit le texte ». Le dernier chapitre, consacré aux rapports entre la littérature et l'histoire, pose la question du matérialisme historique comme méthode d'analyse des productions culturelles. Il s'agit essentiellement d'analyser comment le social s'inscrit dans le texte et par quelles voies. Ici, Edmond Cros propose une vision panoramique des différentes théories successives, du structuralisme génétique à Bakhtine.

4 La troisième partie, intitulée «Sujet culturel et production culturelle » est constituée par 5 études de l'auteur sur l'émergence de la figure du vieux chrétien à la fin du Moyen Âge espagnol, le sujet culturel colonial et l'altérité, le sujet culturel et l'idéal du Moi dans la nouvelle de l'Abencerrage, la mise en scène du sujet culturel dans un portrait d'auteur, le sujet culturel dépouillé de son histoire dans un roman latino-américain contemporain. Une dernière étude d'Annie Buissière sur le sujet culturel dans le roman post-franquiste clôt cette partie.

5 Une courte dernière partie "Le texte culturel, théories et applications ", propose une réflexion autour de ce dernier concept. Une brève définition ouvre l'ensemble, rappelant que le texte culturel «n'a pas de véritable vie autonome ». " Il n'existe que reproduit dans un objet culturel sous la forme d'une organisation sémiotique sousjacente qui ne se donne que fragmentairement à voir, dans le texte émergé, par le biais de traces imperceptibles » La présentation est suivie d'une étude sur Viridiana de Luis Buñuel et la rémanence du Petit Chaperon rouge et de Cendrillon dans le film. L'ouvrage se termine par une étude du même type sur Femmes au bord de la crise de nerf de Pedro Almodóvar.

On retrouvera les réflexions et mises au point les plus récentes d'Edmond Cros sur un site très actif consacré à la sociocritique http://www.sociocritique.fr 